\title{
COMMUTING WELL-BOUNDED OPERATORS ON HILBERT SPACES
}

\author{
by T. A. GILLESPIE $\dagger$ \\ (Received 5th February 1975)
}

\section{Introduction}

A bounded linear operator $T$ on a complex reflexive Banach space is said to be well-bounded if it is possible to choose a compact interval $J=[a, b]$ and a positive constant $M$ such that

$$
\|p(T)\| \leqq M\left\{\|p\|_{J}+\underset{J}{\operatorname{var}} p\right\}
$$

for every complex polynomial $p$, where $\|p\|_{J}$ denotes sup $\{|p(t)|: t \in J\}$. Such operators were introduced and first studied by Smart (4). They are of interest principally because they admit (and in fact are characterised by) an integral representation similar to, but in general weaker than, the integral representation of a self-adjoint operator on a Hilbert space. (See (2) and (4) for details.) It is easily seen, by verifying (1) directly, that $T$ is well-bounded if it is a scalartype spectral operator with real spectrum.

It is known that the sum and product of two commuting scalar-type spectral operators on a Hilbert space are themselves scalar-type spectral operators. (See the proof of Corollary XV.6.5 on p. 1948 of (1).) The purpose of this note is to show that, in contrast, neither the sum nor the product of two commuting well-bounded operators on a Hilbert space is necessarily well-bounded. On the other hand, it is shown in a positive direction that, if one of the two wellbounded operators is in fact a scalar-type spectral operator, then their sum and product are well-bounded.

\section{Notation}

Given a complex Hilbert space $H, B(H)$ denotes the set of all bounded linear operators on $H, I$ is the identity operator on $H$, and $\sigma(T)$ is the spectrum of an element $T$ of $B(H)$. The strong operator topology on $B(H)$ is denoted by $s$ and, given a sequence $\left\{T_{n}\right\}$ in $B(H)$, we write

$$
T=s-\sum_{n=1}^{\infty} T_{n}
$$

to mean that the series $\sum_{n=1}^{\infty} T_{n}$ is s-convergent in $B(H)$, with sum $T . R$ is the

$\dagger$ This research was undertaken at Indiana University with the support of the Science Research Council. 
set of real numbers and $\mathscr{P}$ is the set of all polynomials in a single variable with complex coefficients.

\section{An example}

The example of two commuting well-bounded operators $S$ and $T$ on a Hilbert space such that neither $S+T$ nor $S T$ is well-bounded is based on Example 14.5 on pp. $429-431$ of (3). In that example, sequences $\left\{x_{n}\right\}$ and $\left\{y_{n}\right\}$ in the space $l_{2}$ are constructed having the following properties:

(a) $\left\{x_{n}\right\}$ is a (conditional) basis of $l_{2}$;

(b) $\left(x_{n}, y_{m}\right)=\delta_{n m}$ for $n, m=1,2, \ldots$;

(c) $\left(e_{2 n-1}, y_{2 m-1}\right)=\delta_{n m}$ for $n, m=1,2, \ldots$;

(d) $\left\|\sum_{j=1}^{n} n^{-\frac{1}{2}} x_{2 \jmath-1}\right\| \rightarrow \infty$ as $n \rightarrow \infty$ (see (3), p. 431, line 3);

where $e_{1}, e_{2}, \ldots$ is the usual orthonormal basis of $l_{2}$ and $\delta_{n m}$ is the Kronecker delta.

It follows from $(b)$ that the coefficient functional associated with the element $x_{m}$ of the basis $\left\{x_{n}\right\}$ is given by

$$
x \rightarrow\left(x, y_{m}\right) \quad\left(x \in l_{2}\right) .
$$

Defining $P_{n} \in B\left(l_{2}\right)$ by

$$
P_{n} x=\left(x, y_{n}\right) x_{n} \quad\left(x \in l_{2}\right)
$$

for $n=1,2, \ldots$, it is thus seen that each $P_{n}$ is a projection, $P_{n} P_{m}=0(n \neq m)$, and

$$
I=s-\sum_{n=1}^{\infty} P_{n} \text {. }
$$

Also, if $z_{n}=n^{-\frac{1}{2}}\left(e_{1}+e_{3}+\ldots+e_{2 n-1}\right)$, then $z_{n}$ is a unit vector and so, using (c),

$$
\left\|\sum_{j=1}^{n} P_{2 j-1}\right\| \geqq\left\|\sum_{j=1}^{n} P_{2 j-1} z_{n}\right\|=\left\|\sum_{j=1}^{n} n^{-\frac{1}{2}} x_{2 j-1}\right\| \text {. }
$$

Hence, by $(d)$,

$$
\left\|\sum_{j=1}^{n} P_{2 j-1}\right\| \rightarrow \infty \quad \text { as } n \rightarrow \infty \text {. }
$$

By (2) and the principle of uniform boundedness, the partial sums of the series $\sum_{n=1}^{\infty} P_{n}$ are bounded in norm, and so (3) implies that

$$
\left\|\sum_{j=1}^{n} P_{2 j}\right\| \rightarrow \infty \quad \text { as } n \rightarrow \infty \text {. }
$$

Lemma 1. Let $\left\{\lambda_{n}\right\}$ be a monotonic bounded sequence in $R$. Then the series $\sum_{n=1}^{\infty} \lambda_{n} P_{n}$ converges strongly in $B\left(l_{2}\right)$ and its sum is a well-bounded operator. 
Proof. Setting $Q_{n}=\sum_{j=1}^{n} P_{j}$, Abel's summation lemma gives

$$
\sum_{j=1}^{n} \lambda_{j} P_{j}=\lambda_{n+1} Q_{n}+\sum_{j=1}^{n}\left(\lambda_{j}-\lambda_{j+1}\right) Q_{j}
$$

for $n=1,2, \ldots$. Since $\left\{\lambda_{n}\right\}$ is convergent and $Q_{n} \rightarrow I$ strongly, the sequence $\left\{\lambda_{n+1} Q_{n}\right\}$ converges strongly in $B\left(l_{2}\right)$. As remarked above, the sequence $\left\{Q_{n}\right\}$ is bounded in norm. This, together with the monotonicity and boundedness of $\left\{\lambda_{n}\right\}$, readily implies that $\sum_{j=1}^{\infty}\left(\lambda_{j}-\lambda_{j+1}\right) Q_{j}$ is norm convergent. Thus the right-hand side of (5) $s$-converges in $B\left(l_{2}\right)$ as $n \rightarrow \infty$, and so $\sum_{n=1}^{\infty} \lambda_{n} P_{n}$ is $s$-convergent.

Let $T=s-\sum_{n=1}^{\infty} \lambda_{n} P_{n}$, let $M$ be a positive constant such that

$$
\left\|Q_{n}\right\| \leqq M \quad(n=1,2, \ldots)
$$

let $J$ be a compact interval containing $\left\{\lambda_{n}: n=1,2, \ldots\right\}$, and let $p \in \mathscr{P}$. Since $P_{n}^{2}=P_{n}$ and $P_{n} P_{m}=0(n \neq m)$,

$$
p\left(\sum_{j=1}^{n} \lambda_{j} P_{j}\right)=\sum_{j=1}^{n} p\left(\lambda_{j}\right) P_{j}
$$

and, by applying Abel's lemma again, this gives

Therefore

$$
p\left(\sum_{j=1}^{n} \lambda_{j} P_{j}\right)=p\left(\lambda_{n+1}\right) Q_{n}+\sum_{j=1}^{n}\left(p\left(\lambda_{j}\right)-p\left(\lambda_{j+1}\right)\right) Q_{j} .
$$

$$
\begin{aligned}
\left\|p\left(\sum_{j=1}^{n} \lambda_{j} P_{j}\right)\right\| & \leqq M\left\{\left|p\left(\lambda_{n+1}\right)\right|+\sum_{j=1}^{n}\left|p\left(\lambda_{j}\right)-p\left(\lambda_{j+1}\right)\right|\right\} \\
& \leqq M\left\{\|p\|_{J}+\underset{J}{\operatorname{var} p\}}\right.
\end{aligned}
$$

for $n=1,2, \ldots$. Letting $n \rightarrow \infty$, it follows that

$$
\|p(T)\| \leqq M\left\{\|p\|_{J}+\underset{J}{\operatorname{var}} p\right\},
$$

showing that $T$ is well-bounded and completing the proof.

Define sequences $\left\{\lambda_{n}\right\}$ and $\left\{\mu_{n}\right\}$ by putting

$$
\lambda_{n}=(n+1) / n, \quad \mu_{2 n-1}=\mu_{2 n}=(2 n-1) / 2 n
$$

for $n=1,2, \ldots$, and let

$$
S=s-\sum_{n=1}^{\infty} \lambda_{n} P_{n}, \quad T=s-\sum_{n=1}^{\infty} \mu_{n} P_{n} .
$$


Then $S$ and $T$ are well-bounded operators on $l_{2}$ by Lemma 1, and clearly $S T=T S$. We show that neither $S+T$ nor $S T$ is well-bounded.

Suppose that $S+T$ is well-bounded. Then, by Theorem A (ii) of (4), there is a unique bounded projection $Q$ mapping $l_{2}$ onto the eigenspace

$$
\left\{x \in l_{2}:(S+T) x=2 x\right\}
$$

such that $Q$ belongs to the bicommutant of $S+T$. In particular, $Q P_{n}=P_{n} Q$ for all $n$. Noting that

and that

$$
(S+T) P_{n}=\left(\lambda_{n}+\mu_{n}\right) P_{n} \quad(n=1,2, \ldots)
$$

$$
\lambda_{n}+\mu_{n}=2(n \text { even }), \lambda_{n}+\mu_{n}>2(n \text { odd }),
$$

it is easily verified that

$$
Q P_{n}=P_{n}(n \text { even }), Q P_{n}=0(n \text { odd }) .
$$

Since $I=s-\sum_{n=1}^{\infty} P_{n}$, it follows that

$$
Q=s-\sum_{n=1}^{\infty} Q P_{n}=s-\sum_{n=1}^{\infty} P_{2 n} .
$$

Therefore the partial sums of the series $\sum_{n=1}^{\infty} P_{2 n}$ are bounded in norm by the principle of uniform boundedness, contradicting (4). Hence $S+T$ is not well-bounded.

Since $\lambda_{n} \mu_{n}=1$ ( $n$ odd) and $\lambda_{n} \mu_{n}<1$ ( $n$ even), a similar argument shows that $S T$ is not well-bounded, for otherwise (3) would be contradicted.

\section{A theorem}

Throughout this section, let $H$ be a complex Hilbert space. Given a wellbounded operator $T$ on $H$, we say that $T$ is implemented by $(M, J)$ if $M$ is a positive constant and $J$ a compact interval such that (1) holds for all $p \in \mathscr{P}$.

Lemma 2. Let $T$ be a well-bounded operator on $H$ implemented by $(M, J)$, let $\lambda \in R$, and let $K$ be a compact interval such that $\lambda+t, \lambda t \in K$ for all $t \in J$. Then $\lambda I+T$ and $\lambda T$ are well-bounded operators, and are implemented by $(M, K)$.

Proof. This is a straightforward application of the definition of wellboundedness.

Lemma 3. Let $T$ be a well-bounded operator on $H$ implemented by $(M, J)$, let $S$ be a self-adjoint operator on $H$ with finite spectrum such that $S T=T S$, and let $K$ be a compact interval such that $\lambda+t, \lambda t \in K$ whenever $t \in J$ and $\lambda \in \sigma(S)$. Then $S+T$ and $S T$ are well-bounded operators, and are implemented by $(M, K)$. 
Proof. Let $\sigma(S)=\left\{\lambda_{1}, \ldots, \lambda_{n}\right\}$, where $\lambda_{1}, \ldots, \lambda_{n}$ are distinct. Then there are self-adjoint projections $P_{1}, \ldots, P_{n}$ in $B(H)$ such that $I=\sum_{j=1}^{n} P_{j}$,

$$
P_{i} P_{j}=0(i \neq j)
$$

and $S=\sum_{j=1}^{n} \lambda_{j} P_{j}$

Let $p \in \mathscr{P}$. Since $T$ commutes with $S, T P_{j}=P_{j} T$ for $j=1, \ldots, n$, and hence

$$
p(S+T)=\cdot \sum_{j=1}^{n} p\left(\lambda_{j} I+T\right) P_{j} .
$$

Noting that $p\left(\lambda_{j} I+T\right) P_{j} H \subset P_{j} H$ for $j=1, \ldots, n$, it is thus seen that

$$
\|p(S+T) x\|^{2}=\sum_{j=1}^{n}\left\|p\left(\lambda_{j} I+T\right) P_{j} x\right\|^{2}
$$

for $x \in H$. By Lemma 2,

$$
\left\|p\left(\lambda_{j} I+T\right) P_{j} x\right\|^{2} \leqq M^{2}\left\{\|p\|_{K}+\underset{K}{\operatorname{var}} p\right\}^{2}\left\|P_{j} x\right\|^{2}
$$

for $x \in H$ and $j=1, \ldots, n$. Hence, for $x \in H$,

$$
\begin{aligned}
\|p(S+T) x\|^{2} & \leqq M^{2}\left\{\|p\|_{K}+\underset{K}{\operatorname{var}} p\right\}^{2} \sum_{j=1}^{n}\left\|P_{j} x\right\|^{2} \\
& =M^{2}\left\{\|p\|_{K}+\underset{K}{\operatorname{var}} p\right\}^{2}\|x\|^{2},
\end{aligned}
$$

giving the required result for $S+T$. The result for $S T$ is proved similarly.

Theorem. Let $T$ be a well-bounded operator on $H$, and let $S$ be a scalar-type spectral operator on $H$ with real spectrum such that $S T=T S$. Then $S+T$ and ST are well-bounded.

Proof. Since $S$ has real spectrum, Theorem XV.6.4 on p. 1947 of (1) implies that $S$ is similar to a self-adjoint operator. Furthermore, it is easily seen that the property of well-boundedness is invariant under a similarity transformation. Consequently, we may assume without loss of generality that $S$ is self-adjoint.

Let $T$ be implemented by $(M, J)$ and let $K$ be a compact interval such that $\lambda+t, \lambda t \in K$ whenever $\lambda \in \sigma(S)$ and $t \in J$. By the spectral theorem,

$$
S=\int \lambda F(d \lambda)
$$

for some spectral measure $F(\cdot)$ on $H$ with support $\sigma(S)$, and $T$ commutes with $F(\cdot)$ since it commutes with $S((\mathbf{1})$, Corollary XV.3.7, p. 1935). By approximating the integrand in this integral by step functions, a standard argument gives the existence of a sequence $\left\{S_{n}\right\}$ of self-adjoint operators, each with finite spectrum contained in $\sigma(S)$ and each commuting with $T$, such that $\left\|S-S_{n}\right\| \rightarrow 0$. 
Let $p \in \mathscr{P}$. By Lemma 3,

$$
\left\|p\left(S_{n}+T\right)\right\| \leqq M\left\{\|p\|_{K}+\underset{\mathbf{x}}{\operatorname{var}} p\right\}
$$

for all $n$. Letting $n \rightarrow \infty$, we obtain

$$
\|p(S+T)\| \leqq M\left\{\|p\|_{K}+\underset{K}{\operatorname{var}} p\right\},
$$

which shows that $S+T$ is well-bounded. The well-boundedness of $S T$ is obtained similarly.

\section{REFERENCES}

(1) N. Dunford and J. T. SChWARTZ, Linear operators, Part III: Spectral operators (Wiley-Interscience, New York, 1971).

(2) J. R. RINGrose, On well-bounded operators, J. Austral. Math. Soc. 1 (1959/60), 334-343.

(3) I. Singer, Bases in Banach spaces I (Springer-Verlag, Berlin, 1970).

(4) D. R. SMART, Conditionally convergent spectral expansions, J. Austral. Math. Soc. $1(1959 / 60), 319-333$.

UNIVERSITY OF EDINBURGH 